\title{
SPECIFIC ANTIPNEUMOCOCCAL IMMUNITY IN RELATION TO THE CHEMOTHERAPY OF PNEUMONIA ${ }^{1,2}$
}

\author{
By WILliam S. TILlETT, with the assistance of MARGARET J. CAMBIER \\ AND HAROLD DUNN
}

(From the Department of Medicine, New York University College of Medicine, and the Third Medical Division of Bellevue Hospital, New York)

(Received for publication September 29, 1941)

The findings recorded in this report are concerned with the effect of chemotherapy on the occurrence of type specific pneumococcal antibodies in patients suffering from pneumonia. The incidence of the development of demonstrable specific immunity has been determined with samples of serum obtained at frequent intervals from patients with pneumonia, who were treated with either sulfapyridine or sulfathiazole. In addition to the quantitative aspects of the immunological information derived from the observations, the serological results have also formed the basis for an analysis of the significance of the presence or absence of measurable specific antibodies, in relation to the outcome of the infection in patients treated with either one drug or the other.

The patients available for the immunological study presented varied clinical courses and suffered from pneumonia of varying degrees of severity. Approximately one-third of the group had bacteremia. Although the majority of them recovered promptly from the infection, some had complicating febrile episodes, with and without bacteremia, and still others died. By correlating the different kinds of clinical courses with the results of the serological tests for specific antibodies, the attempt has been made to reconstruct the course of the disease on the basis of the immunological findings. In elaborating the second phase of the problem, the study has also included clinical and postmortem findings of other patients with pneumonia who were not used for serological study, but who were serviceable in the assay of factors that appeared either to promote or retard recovery, or were associated with fatal termination.

\footnotetext{
1 This study was supported by a grant donated by $\mathrm{Mr}$. Bernard Baruch.

2 Part of the results contained in this article was presented at the annual meeting of the Association of American Physicians, Atlantic City, N. J., May, 1941.
}

Although most of the patients were treated with drugs alone, in a few instances, which will be subsequently noted, therapeutic antipneumococcal serum was also administered.

Wood and Long (1) made observations on the development of type specific antibodies in 12 patients with pneumonia who were treated with sulfapyridine. The antibody response was found to be similar to that of untreated cases of pneumonia. Finland, Spring and Lowell (2) made serological studies on more than 100 pneumonic patients treated with drugs and concluded that the antibody response was comparable to that resulting from spontaneous recovery. They found that protective antibodies rarely developed before the sixth day of disease. Kneeland and Mulliken $(3,4)$ have reported the results which they obtained with type specific precipitin reactions performed with specimens of serum from patients with pneumonia. Kneeland and Mulliken obtained specific precipitin reactions in only 4 cases out of 19 treated with sulfapyridine, whereas, again using the precipitin reaction, they found positive reactions in 16 of 21 patients who had been treated with sulfathiażole.

In experimental studies, Whitby (5), and McIntosh and Whitby (6) observed that mice cured of pneumococcus infections with sulfapyridine, subsequently exhibited active specific immunity against the type of organism used in the therapeutic tests. The latter authors concluded that sulfapyridine in no way altered the development of an immune response. MacLeod (7) described results which emphasized the close parallelism between the dosage of pneumococci amenable to the curative action of sulfapyridine and the dosage of heat killed organisms which induced active immunity. He further demonstrated that when sulfapyridine and antipneumococcus serum were employed simultaneously, recovery of mice could be obtained with combined doses in amounts that individually were ineffective.

Plummer, Liebmann, Solomon, Kammerer, Kalkstein, and Ensworth (8) have reported the results obtained from the comparative treatment of 607 patients with pneumonia. The difference in the fatality rate between the patients receiving drug plus serum as compared with those receiving drug alone was negligible. Dowling, Abernethy, and Hartman (9) have advocated the use of combined treatment in patients past 40 years of age. Bullowa, Osgood, Bukantz, and Brownlee (10) obtained a lower death rate with sulfapyridine alone than with any 
other method. Bukantz, Bullowa, and deGara (11) suggested that determinations of pneumococcal polysaccharide in the blood of patients with pneumonia might serve as an indication for the use of serum.

\section{MATERIALS AND METHODS}

Patients. The cases of pneumonia were selected from those admitted to the Wards of the Third Medical Division of Bellevue Hospital during the fall, winter, and spring of 1939-40 and of 1940-41. Serological tests were limited to infections due to pneumococcus Types I, II, III, V, VII, and VIII.

Serum. Repeated bleedings, usually every 2 to 3 days, were obtained from the patients during their stay in the hospital. The first sample was taken before treatment was begun. Serum, separated in the usual manner with sterile precautions, was regularly employed.

Cultures. Stock cultures of the appropriate pneumococcal types were maintained at maximum degrees of virulence for mice. Culture dilution of $10^{-7}$ was regularly fatal. Sixteen to 18 hour blood-broth cultures were used for injection.

Tests. Passive protection of mice. Two-tenths cc. of serum diluted to $0.5 \mathrm{cc}$., by adding $0.3 \mathrm{cc}$. of physiological salt solution, was regularly employed for injection. Tenfold serial dilutions of the broth cultures of type specific pneumococci were made in sterile broth, so that $0.5 \mathrm{cc}$. of each dilution equaled $0.1 \mathrm{cc}$. to $0.000001 \mathrm{cc}$. of original cultures. The $0.5 \mathrm{cc}$. of diluted serum and the $0.5 \mathrm{cc}$. of dilutions of culture were drawn into the same syringe and injected intraperitoneally into mice. Mice receiving only cultures in dilutions of $10^{-5}$ and $10^{-6}$ were used as virulence controls in each experiment. Routinely, the protective power of sera was tested against 4 dilutions of culture ranging from $10^{-8}$ to $10^{-7}$. In some instances, when mice survived $10^{-8}$, the titration was carried to $10^{-1}$. All mice were observed for 7 days.

In order to exclude the remote possibility that the sulfapyridine or sulfathiazole contained in samples of serum obtained during the period of chemotherapy accounted for the protection of mice, many specimens of sera were tested for curative effect against pneumococci heterologous in type to that infecting the patient. In no instance did heterologous protection occur.

Agglutination. Although tests for the presence of type specific agglutinins were not made with all samples of sera, a sufficient number of titrations were performed to indicate the results in comparison with the protection tests. Five-tenths cc. of broth culture plus 0.5 cc. of undiluted serum constituted a final serum dilution of 1 to 2. Other serum dilutions of 1 to 5,1 to 10,1 to 20 , and 1 to 40 were also employed. Final readings were made macroscopically after the tubes had been incubated in the water bath at $37.5^{\circ} \mathrm{C}$. for 2 hours and allowed to stand in the icebox overnight. Type specific agglutination was deemed to be present only when a disc or coarse granulation, characteristic of type specific pneumococcal antigen-antibody reactions, developed.
Two hundred and seventy-five patients have been treated during the 2 year period of study. Twenty-five have died (9.0 per cent). Six additional patients, admitted in a moribund state, died within 6 to 20 hours of admission. Cases with pneumonitis which clearly terminated some other fatal disease have not been included. Cases of acute lower respiratory infection, which were usually mild and of undetermined etiology, have also been excluded.

Ninety-four patients were used in the serological studies. Thirty-one had bacteremia. Fourteen were considered to have protracted febrile illnesses of clinical significance. Ten of the patients died.

The results of the serological tests are summarized in Table I. The presentation of data is so arranged that results referable to the frequency of development of type specific antibodies, and to the approximate time in the course of the disease at which they became demonstrable, are indicated in two separate columns under "Type specific antibodies." Additional columns of tabulation indicate the time of the first positive test in relation to the duration of chemotherapy. The latter arrangement has been made in order to estimate the significance of demonstrable excess antibodies with respect to the outcome of the infection. The bearing of these findings on problems relating to clinical events and the immunological states of the patients will be subsequently discussed.

Frequency of development of type specific antibodies. From the data in Table I, it may be seen that 65 (69 per cent) of the 94 patients developed specific antibodies, the excess of which was sufficiently great to confer passive protection on mice. Among the 84 patients who recovered, 59 (70 per cent) possessed demonstrable serological immunity; among 10 patients who died, 6 (60 per cent) gave positive tests.

Separating the cases of Type III pneumococcus infection, there were, among the other types, 58 ( 80 per cent) instances of the appearance of antibodies among 72 cases. Of the cases of Type III pneumococcus pneumonia, only 8 (36 per cent) out of 22 produced effective passive protection. Serological studies in Type III pneumococcus pneumonia, as well as experimental observations, have uniformly indicated the poor specific antigenicity of Type III pneumococci. The findings in patients receiving chemotherapy are in accord 
with the other studies concerning the formation of antibodies evoked by Type III pneumococci.

It may also be noted from Table I that among the 31 bacteremic patients, 16 (51 per cent) possessed demonstrable excess antibodies, whereas of the 63 non-bacteremic patients, 49 ( 77 per cent) were found to develop measurable type specific immunity. Whether or not the greater amount of antigen present in association with the bacteremia combined with antibody so that the excess was reduced to an unmeasurable minimum, or whether the antibody production was less under the condi-

TABLE I

Occurrence of type specific antibodies in patients with pneumonia treated with sulfapyridine or sulfathiazole

\begin{tabular}{|c|c|c|c|c|c|c|c|c|c|c|}
\hline \multirow{2}{*}{$\begin{array}{c}\text { Pneumo- } \\
\text { coccus } \\
\text { types }\end{array}$} & \multicolumn{2}{|c|}{ Number of cases } & \multicolumn{2}{|c|}{ Bacteremia } & \multicolumn{2}{|c|}{ Type specific antibodies } & \multicolumn{4}{|c|}{$\begin{array}{l}\text { Appearance of antibodies in relation to } \\
\text { duration of therapy }\end{array}$} \\
\hline & Recovered & Died & + & 一 & $\begin{array}{c}\begin{array}{c}\text { Passive } \\
\text { protection } \\
+\end{array} \\
\text {. }\end{array}$ & $\begin{array}{c}\text { Day of disease } \\
\text { 1st positive } \\
\text { test }\end{array}$ & Before & $\begin{array}{c}\text { To 3rd } \\
\text { hospital } \\
\text { day } \ddagger\end{array}$ & "Late" & None \\
\hline \multirow{3}{*}{ I } & & & 5 & & 3 & \multirow{3}{*}{$\begin{array}{l}\text { 6th to } 12 \text { th } \\
\text { av.: } 9 \text { th }\end{array}$} & & 1 & 2 & 2 \\
\hline & -1 & & & 16 & 15 & & 2 & 5 & 8 & 1 \\
\hline & & 0 & & & & & & & & \\
\hline \multirow{4}{*}{ II } & \multirow{2}{*}{28} & & 11 & & 4 & \multirow{4}{*}{$\begin{array}{l}\text { 5th to } 13 \text { th } \\
\text { av.: } 8 \text { th }\end{array}$} & 1 & & 3 & 7 \\
\hline & & & & 17 & 15 & & 3 & 4 & 8 & 2 \\
\hline & & \multirow{2}{*}{$3^{*}$} & 3 & & 0 & & & & & $3^{*}$ \\
\hline & & & & 0 & & & & & & \\
\hline \multirow{4}{*}{ III } & 18 & & 2 & & 0 & \multirow{4}{*}{$\begin{array}{l}\text { 10th to } 12 \text { th } \\
7 \text { th: } 1 \text { case }\end{array}$} & & & & \\
\hline & & \multirow{3}{*}{4} & & 16 & 6 & & & 2 & 4 & 10 \\
\hline & & & 1 & & 1 & & $1 \dagger$ & & & \\
\hline & & & & 3 & 2 & & & & 2 & 1 \\
\hline \multirow{3}{*}{ V } & 5 & & 2 & & 2 & \multirow{3}{*}{$\begin{array}{r}\text { 7th to } 10 \text { th } \\
\text { av.: } 8 \text { th }\end{array}$} & & & 2 & \\
\hline & & & & 3 & 3 & & & & & \\
\hline & & 0 & & & & & 1 & 2 & & \\
\hline \multirow{4}{*}{ VII } & \multirow{2}{*}{9} & & 3 & & 3 & \multirow{4}{*}{$\begin{array}{l}5 \text { th to } 9 \text { th } \\
\text { 15th: } 1 \text { case }\end{array}$} & & 1 & 2 & \\
\hline & & & & 6 & 6 & & 5 & 1 & & \\
\hline & & \multirow{2}{*}{3} & 2 & & 2 & & 1 & 1 & & \\
\hline & & & & 1 & 1 & & 1 & & & \\
\hline \multirow{3}{*}{ VIII } & 2 & & 2 & & 1 & \multirow{3}{*}{ 13th } & & & 1 & 1 \\
\hline & & & & 1 & 1 & & & 1 & & \\
\hline & & 0 & & & & & & & & \\
\hline Total & 84 & 10 & 31 & 63 & 65 & & 15 & 18 & 32 & 29 \\
\hline
\end{tabular}

* Patients died in less than 24 hours after admission.

t Italics indicate fatal cases.

I Includes cases in which 1st test was negative, but passive protection obtained on 2nd or 3rd hospital day. "Late" signifies the presence of passive protective power after the 3rd hospital day. 
tions of excess antigen, has not been determined. The difference in the frequency of excess antibodies between the bacteremic and non-bacteremic group appears to be definite.

Approximate time of appearance of antibodies. In view of the fact that samples of blood were taken every 2 to 3 days instead of daily, the results listed in Table I under "Day of disease of 1st positive test" represent the approximate time at which the immune response was first demonstrable. From averaging all of the data, the protective power of the sera was found to appear in the majority of instances on about the 7th to 10th day. In a few cases, positive tests were not apparent until the 12th to 13th day. An exception to the general results may be noted in the cases of Type III pneumococcus. infection, and also in 2 of the 3 cases of pneumococcus Type VIII pneumonia, in which the development of sufficient antibody to afford passive protection to mice was regularly delayed until the 10th to 13th day.

Titre of specific antibodies. Each sample of serum was regularly tested in mice against $10^{-8}$ dilution of culture which corresponded to 10,000 minimal lethal doses of a virulent strain of the homologous type. With sera obtained from cases of infection due to Types I, II, V, and VII pneumococci, the titre of specific immunity was sufficiently high in the greatest number of instances to afford protection against the greatest dose used in the routine tests. Tests with greater amounts of culture were not done frequently enough to determine the maximum amount of protective antibodies which were present. However, the constancy of the results has indicated that the quantitative production of antibodies by patients was not significantly impaired by chemotherapy. In the patients with Type III pneumococcus pneumonia, as well as in the 3 patients with Type VIII pneumococcus pneumonia, the protective power of sera did not exceed $10^{-4}$ dilution of culture.

Type specific agglutination. Type specific agglutinins were found to be present in 44 (59 per cent) of the 74 sera that were tested. In a number of specimens, the protective power of serum was demonstrable but agglutination did not occur. The apparent difference in the results obtained with the 2 tests is dependent upon the fact that the passive protection of mice is a more delicate method of determining the presence of type specific antipneumococcal antibodies than is the agglutination reaction. Consequently the presence of protective capacity in the absence of agglutination is an expected finding which is dependent upon the quantity of specific immune principles in the samples of sera.

Time of appearance of demonstrable antibodies during the course of chemotherapy. The data in the columns under the heading "Appearance of antibodies in relation to duration of therapy" are arranged for the purpose of analyzing the incidence of demonstrable type specific immunity in relation to the outcome of drug treatment. From the results recorded under the heading "Before," it may be noted that in 15 patients, the samples of serum obtained on the day of admission-before treatment was begun-contained specific protective antibodies. In 18 additional patients, specific antibodies had become demonstrable by the 3rd day after admission. This group has been separated because of the fact that the first 3 days most often constitute the "crucial" period of therapy. During this period, essential recovery or marked improvement may occur; a continued illness may persist; or the patient may become progressively worse. In analyzing the factors that influence the outcome of the infection, therefore, it is a matter of particular interest to evaluate the immunological status of the patients during the early phase of treatment. Combining the cases in which specific antibodies were present before treatment was instituted with the cases in which protective power became evident by the 3rd day of hospitalization, it may be noted that in 33 instances excess antibodies were present in the early phase of chemotherapy.

Under the column headed "Late," in Table I, there is a list of 32 cases in which measurable amounts of free circulating specific antibodies did not appear until after the 3rd hospital day. In some instances, chemotherapy was being continued when the protective action of the serum became positive, and, in other instances, sulfonamide treatment had ceased before specific immunity was demonstrable. Since, however, the extent of therapy was determined solely on the basis of the clinical condition of the patient, the serological findings did not influence the duration of sulfonamide administration. Consequently, in this 
group, a relationship of the time of appearance of positive immunological findings to the response to chemotherapy was not clearly defined.

In the last column of Table I, there are listed 29 patients in whose sera type specific antibodies were not demonstrable at any time during the course of the illness. In connection with the implied immunological difference between the patients with and without demonstrable antibodies, it is important to re-emphasize the fact that the failure of a sample of serum from a patient to protect mice by passive immunization may not be final proof of the total absence of specific antibodies. It has been demonstrated experimentally that effective active immunity may be developed, even though the serum of the animal does not afford protection to other animals. Consequently the difference between the patients with protective antibodies and those without them may be quantitative rather than entirely qualitative.

In summarizing the findings that deal with the time of appearance of antibodies in relation to period of drug treatment, the results demonstrate that in 33 of the cases ( 35 per cent), specific immunity was present during the first 3 days of treatment, either at the time of admission of the patient (15 instances) or within the early period of hospitalization (18 instances). In 32 patients (34 per cent), excess antibodies became demonstrable at varying periods later than the 3 rd day of hospitalization. In 29 patients (31 per cent), the various samples of sera, which were tested, failed in all instances to confer passive protection.

The fact that the cases which developed free circulating antibodies are scattered essentially evenly among the 3 groups of patients indicates the absence of definite correlation between the period of administration of sulfonamide drugs and the functioning of the mechanism of antibody production.

Although the selection of cases included in this study was not determined by the type of drug (sulfapyridine or sufathiazole) used for treatment, an analysis of the 2 groups reveals the fact that 62 of the patients received sulfapyridine and 29 received sulfathiazole. The patients dying in less than 24 hours after admission are omitted. The results obtained following the 2 different medications are recorded in Table II according to pneumococcus types causing infection and the incidence of immune response as demonstrated by the protective capacity of sera for mice.

TABLE II

\begin{tabular}{c|c|c|c|c|c|c}
\hline $\begin{array}{c}\text { Pneumo- } \\
\text { coccus } \\
\text { types }\end{array}$ & $\begin{array}{c}\text { Num- } \\
\text { ber of } \\
\text { cases }\end{array}$ & Sulfapyridine & $\begin{array}{c}\text { Num- } \\
\text { ber of } \\
\text { cases }\end{array}$ & Sulfathiazole \\
\hline & & \multicolumn{3}{|c|}{ Specific antibodies (Passive protection) } \\
\hline & & Present & Absent & & Present & Absent \\
\hline II & 16 & 12 & 4 & 5 & 5 & 0 \\
III & 16 & 12 & 4 & 12 & 8 & 4 \\
V & 13 & 6 & 7 & 9 & 3 & 6 \\
VII & 10 & 10 & 0 & 1 & 1 & 0 \\
VIII & 3 & 2 & 1 & 2 & 2 & 0 \\
Total & 62 & 46 & 16 & 29 & 19 & 10 \\
& & 4 & 0 & 0 & 0 \\
\hline
\end{tabular}

From the tabulated data it is seen that of the 62 patients treated with sulfapyridine, 46 ( 74 per cent) developed demonstrable type specific antibodies, and that the sera from 19 (65 per cent) of the 29 patients receiving sulfathiazole afforded similar protection. In view of the relatively small number of patients included in each group, the difference in the results does not seem significant.

In considering the clinical courses of the patients who were used for serological observations, recovery was uneventful in 70 . Fifty-five ( 75 per cent) developed type specific antibodies; in 15, none were detected. The nature of the recovery just mentioned indicates that there was no apparent difference in the progress of the patients possessing demonstrable antibodies and the others.

Of the 14 patients whose recovery was significantly delayed because of protracted febrile illness, in 5, type specific antibodies were demonstrable early after hospitalization, in 5 others, they appeared during the late period, and in 4 , none were evident up to the time of discharge from the hospital. The majority of the patients who pursued stormy courses were among those with bacteremia. As stated earlier in the report, 16 (51 per cent) of the 31 patients with bacteremia developed measurable amounts of specific immunity. When the clinical records of the 16 with antibodies and the 15 without were compared, no clear-cut differentiation could be made. There were certain clinical impressions that in the bacteremic patients without demonstrable antibodies, recovery may in some instances have been retarded or 
resolution may have been delayed to a greater degree than in bacteremic patients who developed specific antibodies. However, in view of the variables in the factors of pneumonia which may be referable, on the one hand, to intensity of infection or the nature of the complications, such as empyema or sterile pleural effusion, or, on the other, may depend upon the general physical status of the hosts of the infection, it has not been possible, among the bacteremic patients, to account for differences in clinical behaviour by an analysis of objective data obtained by the serological methods used in the present study.

Individual charts are presented which illustrate different types of courses in relation to the immunological finding.

Figure 1 is that of a patient who had, on admission, primary pneumonia and bacteremia due to pneumococcus Type I. He came to the hospital on the 2nd day of the disease. Because of clinical evidence of rapid improvement, drug therapy was deliberately stopped, after 13 grams had been given in 30 hours, in order to observe the course in an early case receiving limited therapy. The subsequent rise in fever seemed to represent a relapse of pneumonia, although bacteremia did not return. Permanent cure, following the re-administration of sulfapyridine, appeared to coincide with the appearance of excess antibodies.

Figure 2 is that of a patient who had, on admission, primary pneumonia and bacteremia due to pneumococcus Type II. His progress following chemotherapy was one of continued daily improvement leading to uneventful recovery. No excess antibodies were found in any of the speci-

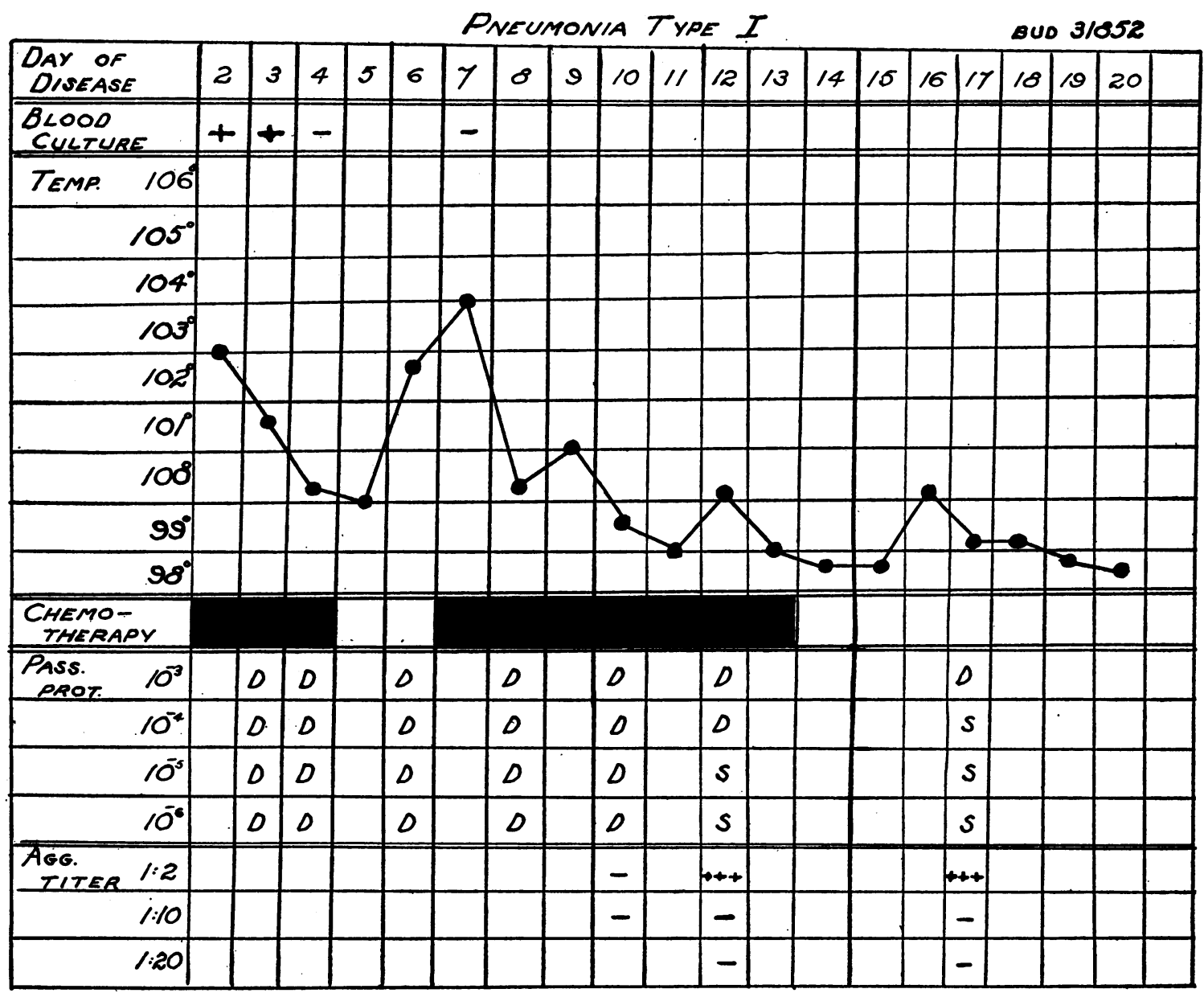

FIG. 1 
Pneumonia Type II

AND 34570

\begin{tabular}{|c|c|c|c|c|c|c|c|c|c|c|c|c|c|c|c|c|c|c|c|c|}
\hline $\begin{array}{l}\text { DaY OF } \\
\text { DLSEASE }\end{array}$ & 4 & 5 & 6 & 7 & 8 & 9 & 10 & 11 & 12 & 13 & 14 & 15 & 16 & 17 & 18 & 19 & 20 & 21 & 22 & 23 \\
\hline $\begin{array}{l}\text { BLOOD } \\
\text { KLTURE }\end{array}$ & + & & 一 & & & & & & & & & & & & & & & & & \\
\hline TEMP. $\quad 106^{\circ}$ & & & & & & & & & & & & & & & & & & & & \\
\hline $105^{\circ}$ & & & & & & & & & & & & & & & & & & & & \\
\hline 104$]$ & & & & & & & & & & & & & & & & & & & & \\
\hline 103 & & & & & & & & & & & & & & & & & & & & \\
\hline 102 & & & & & & & & & & & & & & & & & & & & \\
\hline $100^{\circ}$ & & & & & & & & & & & & & & & & & & & & \\
\hline $100^{\circ}$ & & & & & & & & & & & & & & & & & & & & \\
\hline & & & & & & & & & & & & & & & 8 & & & & & \\
\hline $\begin{array}{l}\text { CHEMO- } \\
\text { IHERAPY }\end{array}$ & & & & & & & & & & & & & & & & & & & & \\
\hline $\begin{array}{l}\text { Pass. } 10^{-3} \\
\text { Pfot. }\end{array}$ & $D$ & & $D$ & & $D$ & & & & & & & & & & $D$ & & & & $D$ & \\
\hline $10^{-4}$ & $D$ & & $D$ & & $D$ & & & & & & & & & & 0 & & & & $D$ & \\
\hline 105 & $D$ & & $D$ & & 0 & & & & & & & & & & 0 & & & & 0 & \\
\hline $10^{-6}$ & $D$ & & $D$ & & $D$ & & & & & & & & & & $D$ & & & & $D$ & \\
\hline $\operatorname{Acos}_{\text {TES }} 1: 2$ & - & & - & & - & & & & & & & & & & - & & & & - & \\
\hline 1:10 & - & & - & & - & - & & & & & & & & - & - & & & & - & \\
\hline
\end{tabular}

FIG. 2

mens of serum. The possible interpretations of the negative serological findings will be subsequently considered.

Figure 3 is that of a patient having pneumonia and bacteremia (pneumococcus Type VII), who had a protracted febrile illness which seemed clinically to be dependent, to a large degree, upon a recrudescence of active pneumonia and pleurisy, without empyema. It is unnecessary to detail the clinical findings save to indicate the fact that type specific antibodies were present before the secondary febrile episode began.

The chart of the 1 st of the 3 bacteremic cases illustrates the clinical course in relation to the immunological mechanism that was frequently encountered. It seems likely that the febrile relapse could have been prevented either by continuing drug therapy longer or by introducing specific antibodies at the beginning of therapy. However, in other patients the clinical-immunological course of events did not follow the same orderly procedure. The selected charts of the 2 other individual cases illustrate on the one hand, instances of progressive recovery following drug therapy without the mediation of demonstrable excess antibodies, and on the other hand, they exemplify conditions under which the presence of type specific antibodies did not in itself prevent certain febrile episodes that were interpreted clinically as being active pneumonic infection.

Fatal cases of pneumonia. The presentation of material has been arranged in order to particularize and to emphasize certain findings that were characteristically associated with death from pneumonia.

Age. Of the 31 fatal cases, including 6 which 


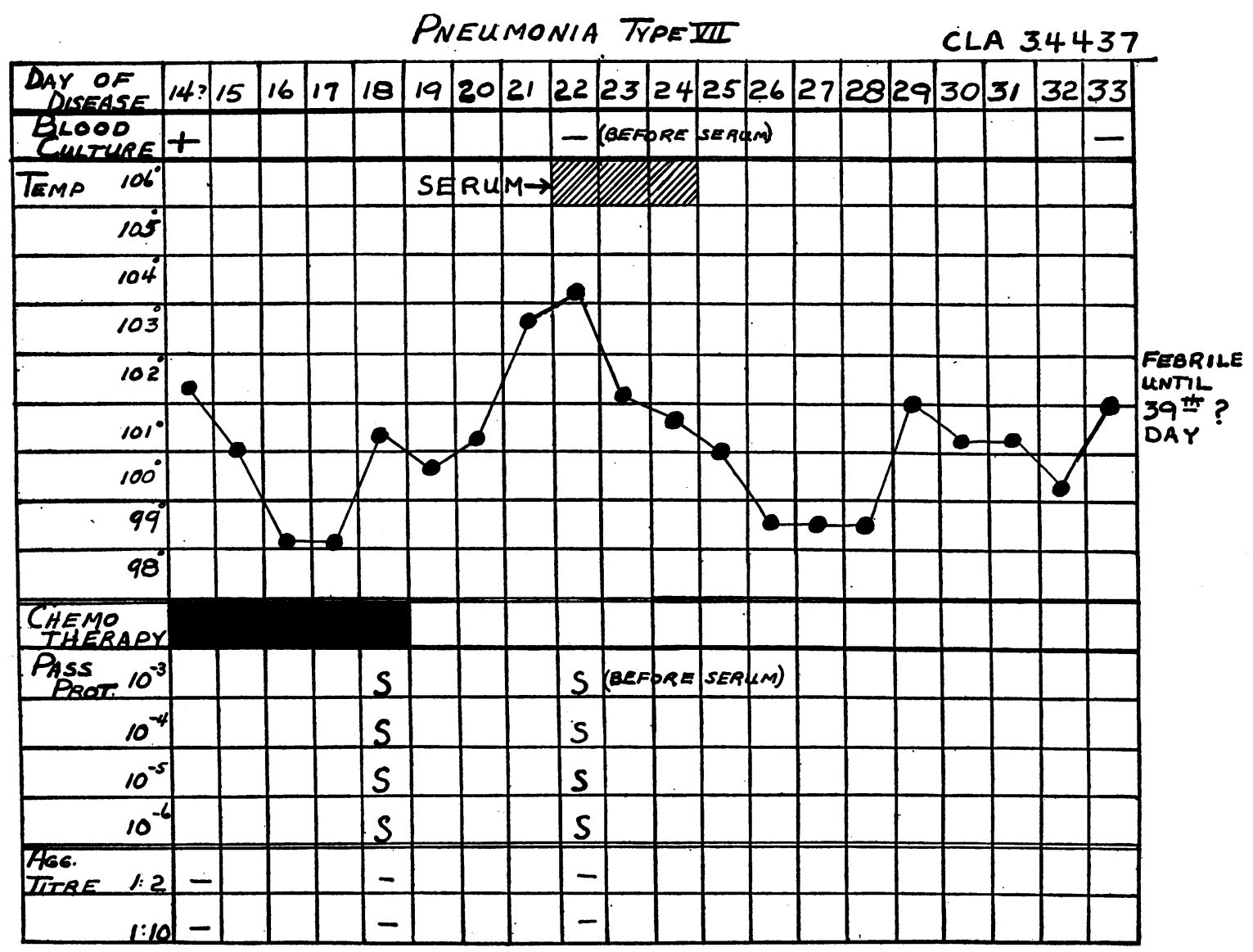

FIG. 3

were moribund on admission, the ages according to decades of life were: 1 between 20 and 30, 2 between 30 and 40,3 between 40 and 50,11 between 50 and 60,10 between 60 and 70,4 between 70 and 80 . Eighty-four per cent were past 50 years of age.

Serological types of infecting pneumococci. The distribution of pneumococcal types was as follows: Type I, 2 cases; Type II, 8 cases; Type III, 8 cases; Type V, 2 cases; Type VII, 4 cases ; Type XIV, 2 cases; and Types VI, XI, XII, XV, and XXV, 1 case each.

Bacteremia. In 21 of the 31 patients, pneumococci were recovered from the blood stream on the initial attempt; in 8 , the first culture was sterile; in 2, no cultures were made. Seventy-two per cent had positive cultures on admission.

In 19 of the group, one or more subsequent cultures were taken. In 15 , the bacteremia disappeared. Each of the 4 patients from whom subse- quent cultures were persistently positive had endocarditis. ${ }^{3}$

Of the 12 cases in which a 2 nd culture was not taken, 6 died on the 1st hospital day (all positive), and the remaining 4 died before the 4 th day ( 3 positive, 1 negative); in 2 , no cultures were taken at any time.

In addition to the statistical data just given, the fatal cases have been further analyzed in an attempt to identify significant factors that were associated with an unfavorable course. For this pur-

${ }^{3}$ During the current winter, 2 patients with lobar pneumonia have been encountered in whom bacteremia persisted for as long as 6 days in spite of adequate chemotherapy. The administration of specific anti-pneumococcal serum therapy was followed in each instance by rapid improvement and prompt sterilization of the blood stream. One of the patients had Type I, and the other Type VIII, pneumococcus pneumonia. Studies of the possible resistance of the strains to the effect of sulfonamides are now being made. 
pose information derived from clinical and pathological sources has been correlated with bacteriological and immunological findings. Utilizing the cases concerning which the findings are complete, a majority may be divided into special groups, depending upon the conditions that appeared to be of special importance in association with unsuccesful treatment.

1. The severity of pneumonia at the time of hospitalization.

2. The presence of metastatic localized infections such as meningitis, endocarditis or pericarditis.

3. The coexistence of other diseases unrelated to pneumonia which were temporarily overshadowed by the manifestations of active infection.

1. Severity of primary pneumonia. On the basis of duration of life, 6 patients were admitted in a moribund state and died within 24 hours of hospitalization, either before treatment was begun or shortly thereafter. Two had meningitis, and 1 also had pericarditis, in addition to pneumonia.

Bacteremia was present in each of the 6 patients.

Immunological results. A single sample of serum was obtained from each of three of the patients. No passive protection was demonstrable.

The patients of this group comprised approximately 20 per cent of the total deaths.

TABLE III

\begin{tabular}{|c|c|c|c|c|c|c|}
\hline \multirow{2}{*}{ Case } & \multirow{2}{*}{ Age } & \multirow{2}{*}{$\begin{array}{l}\text { Pneu- } \\
\text { mococ- } \\
\text { cus } \\
\text { type }\end{array}$} & \multicolumn{2}{|c|}{ Blood cultures } & \multirow{2}{*}{$\begin{array}{c}\text { Specific } \\
\text { antibodies }\end{array}$} & \multirow{2}{*}{$\begin{array}{l}\text { Days in } \\
\text { hospital }\end{array}$} \\
\hline & & & 1st & 2nd & & \\
\hline $\begin{array}{l}\text { L. K. } \\
\text { J. D. } \\
\text { C. M. } \\
\text { R. K. }\end{array}$ & $\begin{array}{l}\mathbf{5 4} \\
56 \\
55 \\
68\end{array}$ & $\begin{array}{r}\text { III } \\
\text { VII } \\
\text { III } \\
\text { I }\end{array}$ & $\begin{array}{l}+ \\
+ \\
+\end{array}$ & $\bar{z}$ & $\begin{array}{c}\text { Serum } *(2) \\
+(2) \\
+(2) \\
\text { No test }\end{array}$ & $\begin{array}{l}2 \\
3 \\
3 \\
2\end{array}$ \\
\hline
\end{tabular}

* Numerals in parentheses indicate hospital day on which serum was taken with which positive protection tests were obtained.

A 2nd group of patients falling within this same category of severe primary pneumonia consisted of patients who lived until the 3rd hospital day, but in spite of therapeutic measures failed to survive. Selected data of bacteriological and immunological interest for 4 of the patients are given in Table III.
The course in this group of patients was characterized by a change of blood cultures from positive to negative and the appearance of type specific antibodies within a short period of time. In spite of the change in the bacteriological immunological balance following chemotherapy, the patients failed to survive. It seems not unlikely that although the extent of the infection, as evidenced by the blood culture, was decreasing during treatment, the initial severity was probably so advanced that even the loss of bacteremia and the acquisition of free circulating antibodies were ineffective in reversing the fatal course.

Six additional patients lived from 4 to 12 days. On clinical grounds, the diagnosis was limited to pneumonia. Their ages ranged from 50 to 74 years.

Bacteremia. In 4, blood cultures were negative throughout the period of hospitalization. In 2, the initial blood culture was positive but subsequent ones were sterile.

No serological tests were performed. No autopsies were obtained.

\section{Metastatic pneumococcal complications.} Meningitis, pericarditis, and endocarditis, singly or in combinations, were present in 10 (32 per cent) of the fatalities. Two died within 24 hours of admission. The remaining 8 survived 3 days or longer, and received intensive therapy.

Five cases had meningitis. Both drug and serum were administered to 2 of the 3 who lived long enough to receive continued treatment.

Three cases had pericarditis. One lived less than 1 day and another died on the 2nd day after admission. The 3rd patient, who lived 4 days, also had meningitis.

Four cases of endocarditis, which were proven by autopsy, and 1 unverified case, were observed among the 275 patients of the present series. It is interesting to note that in subsequent blood cultures, of 4 of the patients with endocarditis, bacteremia either returned after temporary absence or continued to be present for the 10 days' duration of the illness. Although the occurrence of persistent or recurring pneumococcal bacteremia among the cases of pneumonia observed during the 2 year period of this study was small, its association with endocarditis has been sufficiently striking to justify clinical suspicion of endocardial 
involvement when bacteremia is refractory to continued treatment (see footnote number 3 ). No evidence has accrued which makes the present finding particularly unusual, even though endocarditis was proven in 1.4 per cent of the current series. It seems not unlikely that with the marked reduction in the total death rate due to pneumonia, the relative increase in pneumococcal endocarditis, which still remains uniformly fatal, accounts for the statistical difference. Of the $5 \mathrm{pa}$ tients with endocarditis, 2 received serum. The course of the disease in the 5 patients was not different from that previously observed before the advent of chemotherapy .

Bacteremia was present in each of the 10 patients.

Immunological results. Of the cases with complications, no protective antibodies were demonstrated in single samples of serum from 3 . In 4 others, 3 of which received serum therapy, free circulating antibodies were present in at least 1 test. In 3 , no samples of serum were obtained.

3. Coexistence of other diseases unrelated to pneumonia. Seven patients, 22 per cent of the fatalities in the current study, comprised this group. Their illness was characterized by the fact that although lobar pneumonia was the most conspicuous part of the clinical diagnosis on admission, subsequent events, including findings at autopsy, proved that other diseases were of special importance in accounting for the fatal outcome. The patients of this group lived 5 days or longer. Cases in which pneumonitis was manifestly the terminal event in some other disease of maximum severity such as cerebral paralysis or uremia, have been excluded.

It is unnecessary to detail the individual clinical courses. Two of the patients had sudden terminal cardiac episodes during convalescence; in 2 senile female patients, gangrenous cystitis was found at autopsy; 1 patient with extensive anthrasilicosis of 5 lobes had a normal temperature. for the last 6 days of life; 1 patient had an acute lead intoxication demonstrated at autopsy; 1 patient had an obscure and intractable encephalopathy. In each of the above instances, at autopsy, the pneumonia was resolving.

Bacteremia. Three of the patients had bacteremia on admission which disappeared within
48 hours after beginning chemotherapy. In the remaining 4 , all blood cultures were sterile.

Immunological results. In 5 of the patients, repeated serological tests were performed, and in each instance, protection of mice was obtained with samples of serum obtained within the first few days after instituting chemotherapy.

In the foregoing analysis of the fatalities, by combining the cases that clearly belong in the 3 groups, but including only the moribund cases of the severe primary pneumonia group, it may be observed that approximately 74 per cent of the 31 deaths were identified according to special characteristics. In the selected groups, the fact that specific antibodies, when present, did not make up the deficiencies of chemotherapy appeared to be referable to the status of the patients' disease on admission. Of the remaining patients about whom information is not complete enough to warrant definite grouping, it is interesting to note the frequency with which bacteremia disappeared following chemotherapy.

\section{DISCUSSION}

The results described in this report indicate that the administration of sulfonamide drugs (sulfapyridine or sulfathiazole) did not significantly affect either the antigenic integrity of the infecting pneumococci or the immunological responsiveness of patients suffering from pneumonia.

With respect to the frequency of the development of demonstrable antibodies, the time of their appearance during the course of pneumonia, and approximate estimation of titre, the findings did not differ from the general experience derived from previous studies of untreated patients.

However, in spite of the absence of a direct effect of sulfonamide drugs on measured antibody response, indirect effects of chemotherapy on specific antipneumococcal processes were found to occur during the course of pneumonia and to be referable to quantitative alterations in antigenantibody relationships. The studies contained in the present report serve as a basis for an analysis of some of the factors relating to the immunological course.

It has become a well established immunological principle that the antigen-antibody ratio in pneumococcus infections varies during the course of the 
disease from excess antigen (pneumococci and soluble specific substance) and minimum antibody at the onset, to excess antibody and progressively decreasing antigen following recovery. Alterations in the two factors and their bearing upon the course of the disease are referable to changes in the extent of the infection during its progress in relation to antibody production. Specific antipneumococcal serum and the specifically acting sulfonamide drugs both alter the quantitative antigen-antibody relationships, but the result in each instance is obtained by different processes. Therapeutic antiserum operates through primarily increasing the antibody content and secondarily reducing the amount of antigen by opsonization of pneumococci. The use of chemotherapy, on the other hand, is primarily successful by reducing the amount of antigen through the untoward effect of the drugs on the microorganisms. The decrease in antigen effected by the drugs brings about a relative increase in antibodies. It is also interesting to take into account the fact that specific antibodies are used up in the process of combining with pneumococci, whereas experimental studies have not up to the present time indicated that sulfonamide drugs are operative through permanent combination with bacterial cells. Consequently, increments of the drugs that are not inactivated or excreted may remain free for continuous action.

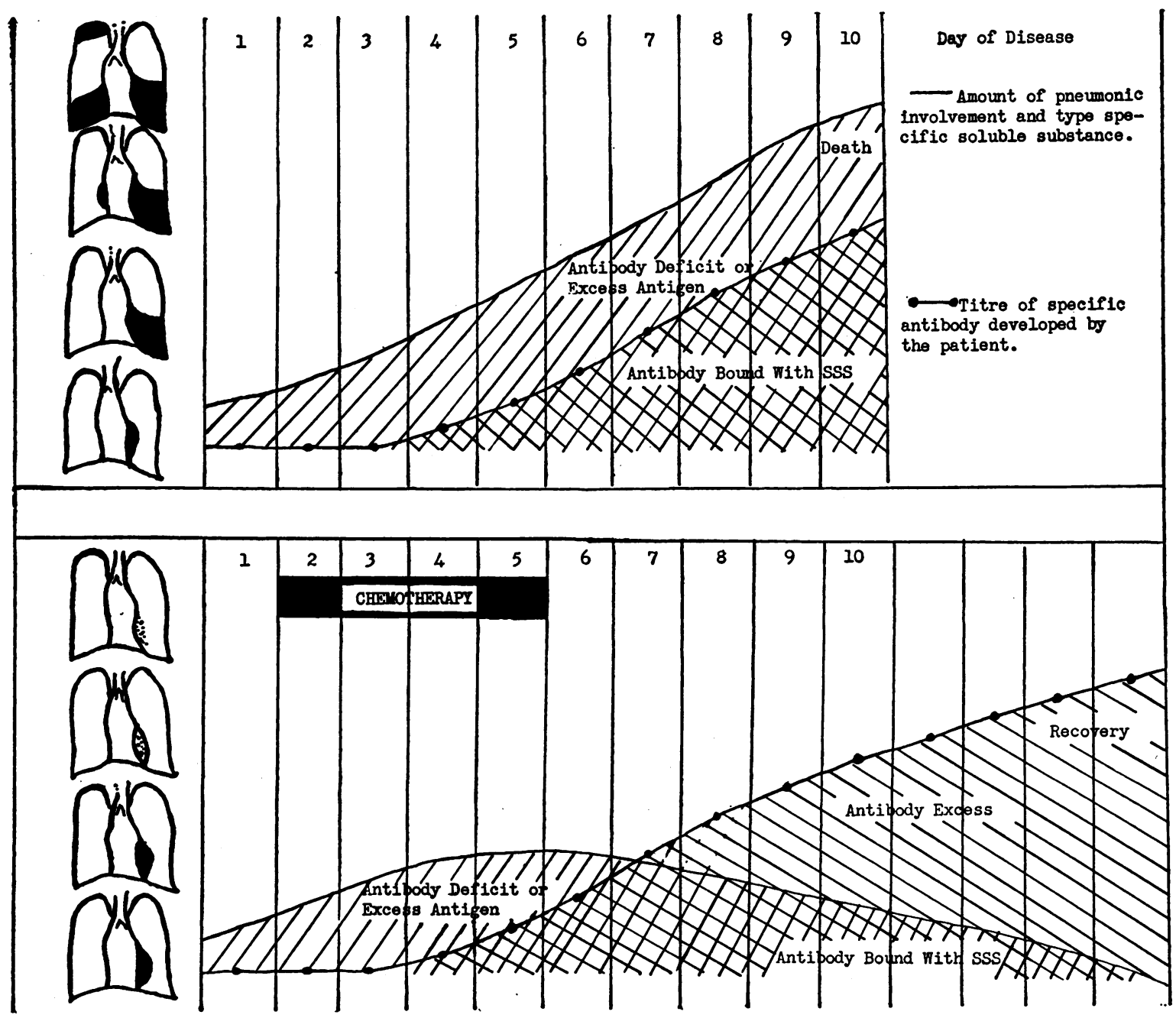

Fig. 4. Theoretical Antigen-Antibody Relationships During Course of Pneumonia in an Untreated Patient and in 1 Receiving Chemotherapy 
The effect of sulfonamide treatment on the antigen-antibody balance in pneumonia is illustrated graphically in Figure 4.

The data obtained from the present study frequently conformed to the course outlined in Figure 4. It appears not unlikely that this chemotherapeutic-immunological relationship occurs in the usual course of uncomplicated pneumonia proceeding to uneventful convalescence. However, with respect to ultimate recovery or death, the presence or absence of demonstrable specific antibodies did not uniformly separate the 2 groups. Exceptions were noted among both the patients who were successfully treated and also among the group who died. There were, for example, among the patients who recovered, a number of instances (24 listed in Table I) in which antibodies were not demonstrated during convalescence. Since, however, only free unbound antibodies are detectable by the methods employed, it is apparent that a negative serological reaction may be due to the fact that, $(a)$ no antibodies have been developed following infection, or $(b)$ all of the antibody was bound to antigen and, as a result, no excess was detectable. In the latter instance, the antibody production by the patient may have been highly effective in neutralizing antigen even though the serological tests were negative. In view of the limitations of the tests, it is not possible to conclude with certainty which of the interpretations is applicable to the findings in the recovered patients whose sera failed to confer passive protection.

In a consideration of the fatal cases, it is apparent that the extensiveness of the pneumococcal infection at the time of admission was frequently of primary importance in determining the outcome. For example, 20 per cent of the deaths occurred within 24 hours of admission. There were among the fatalities also an appreciable number who failed to recover even though specific antibodies were demonstrable in their serum. In correlating the clinical and pathological findings with the immunological results, significant factors associated with death were found to be of such a character that they were not decisively controlled by even the combined effect of chemotherapy and specific antibodies. Approximately 75 per cent of the deaths appeared to belong to the groups described in the body of this report.
Additional factors that may mitigate against satisfactory chemotherapy, such as intolerance of drugs by patients, or infections caused by drugfast strains, or inactivation of sulfonamide compounds by para aminobenzoic acid or similarly acting substances, have not been included in the present study. The importance of their bearing upon optimum therapy with special reference to the use of specific serum therapy is apparent. However, that diverse conditioning elements, not regularly. controlled by specific antibodies, not infrequently account for the unsuccessful use of chemotherapy, has been indicated in the present study.

\section{SUMMARY}

1. Ninety-four patients have been used for serological studies. Thirty-one had bacteremia. Seventy of the total number recovered from pneumonia uneventfully. Fourteen additional patients who also recovered had protracted febrile illness of clinical significance. Ten of the patients died.

During the 2 year period 1939-1941, among 275 patients with pneumonia, who, with a few exceptions, were treated with either sulfapyridine or sulfathiazole alone, 25 (9 per cent) died.

2. Sixty-three (69 per cent) of the 94 patients developed specific antibodies as determined by the passive protection of mice. Fifty-nine (70 per cent) of 84 patients who recovered possessed measurable specific immunity. Six (60 per cent) of 10 patients who died gave positive protection tests.

Eight (36 per cent) of 22 patients with Type III pneumococcus pneumonia produced detectable immunity. Fifty-eight ( 80 per cent) of 72 patients with Types I, II, V, VII, and VIII pneumococci gave positive serological tests.

3. In relation to day of treatment, rather than day of disease, excess antibodies were present in 15 instances before therapy was begun, in 18 instances by the 3rd day of hospitalization, in 32 instances after the 3rd day, and in 29 instances, all tests were negative.

4. A correlation between the clinical course of the disease and the immunological findings was made. Although the majority of the whole group of patients developed measurable amounts of specific immunity, the presence or absence of demonstrable antibodies did not, in some instances, 
decisively separate the patients who satisfactorily responded to chemotherapy from others whose convalescence was delayed.

5. The fatal cases were divided into groups according to the factors that were found to be of special importance in determining the outcome.

6. The quantitative immunological relationships between infecting pneumococci and specific antibodies . which occur during the course of pneumonia treated with sulfonamide drugs have been discussed.

\section{BIBLIOGRAPHY}

1. Wood, W. B., Jr., and Long, P. H., Observations upon the experimental and clinical use of sulfapyridine. III. The mechanism of recovery from pneumococcal pneumonia in patients treated with sulfapyridine. Ann. Int. Med., 1939, 13, 612.

2. Finland, M., Spring, W. C., and Lowell, F. C., Immunological studies on patients with pneumococcic pneumonia treated with sulfapyridine. J. Clin. Invest., 1940, 19, 179.

3. Kneeland, Y., Jr., and Mulliken, B., Antibody formation in cases of lobar pneumonia treated with sulfapyridine. J. Clin. Invest., 1940, 19, 307.

4. Kneeland, Y., Jr., and Mulliken, B., Antibody for- mation in cases of lobar pneumonia treated with sulfathiazole. J. Clin. Invest., 1940, 19, 735.

5. Whitby, L. E. H., Chemotherapy of pneumococcal and other infections with $2-(p$-aminobenzenesulfonamido) pyridine. Lancet, 1938, 1, 1210.

6. McIntosh, J., and Whitby, L. E. H., Mode of action of drugs of the sulfonamide group. Lancet, 1939, $1,431$.

7. MacLeod, C. M., Chemotherapy of pneumococcic pneumonia. J. A. M. A., 1939, 113, 1405.

8. Plummer, N., Liebmann, J., Solomon, J., Kammerer, W. H., Kalkstein, M., and Ensworth, H. K., Chemotherapy versus combined chemotherapy and serum in the treatment of pneumonia. A study of 607 alternated cases. J. A. M. A., 1941, 116, 2366.

9. Dowling, H. F., Abernethy, T. J., and Hartman, C. R., Should serum be used in addition to sulfapyridine in the treatment of pneumococcic pneumonia? J. A. M. A., 1940, 115, 2125.

10. Bullowa, J. G. M., Osgood, E. E., Bukantz, S. C., and Brownlee, I. E., The effect of sulfapyridine alone and with serum on pneumococcic pneumonia and on pneumococcus-infected marrow cultures. Am. J. M. Sc., 1940, 199, 364.

11. Bukantz, S. C., Bullowa, J. G. M., and de Gara, P. F., Detection of free polysaccharide in the blood of pneumococcic pneumonia patients; prognosis and therapy. Proc. Soc. Exper. Biol. and Med., 1939, 41, 250. 